\title{
Comparison of experimental monkey resting state data with large scale
}

neural network simulations

Paulina Dąbrowska', Nicole Voges ${ }^{1}$, Michael von Papen ${ }^{1}$, Alexa Riehle ${ }^{2,1}$, Thomas Brochier', Johanna Senk', Markus Diesmann ${ }^{1.3 .4}$, Sonja Grün ${ }^{1.5}$

Institute of Neuroscience and Medicine (INM-6) and Institute for Advanced Simulation (IAS-6) and JARA Institute Brain Structure-Function Relationships (INM-10), Jülich Research Centre Germany: ${ }^{2}$ Institut de Neurosciences de la Timone (INT) CNRS-Aix-Marseille Université, France: ${ }^{3}$ Department of Psychiatry Psychotherapy and Psychosomatics, Medical Faculty, RWTH Germany; ${ }^{2}$ Institut de Neurosciences de la Timone (INT), CNRS-Aix-Marseille Universite, France; ${ }^{3}$ Department of Psychiatry, Psychotherapy and Psychosomatics, Medical
Aachen University, Germany; ${ }^{4}$ Department of Physics, Faculty 1, RWTH Aachen University, Germany; ${ }^{5}$ Theoretical Systems Neurobiology, RWTH Aachen University, Germany

Contact: p.dabrowska@f-juelich.de

$$
\begin{aligned}
& \text { Aim anc } \\
& \text { To better understand the network activity of macaque motor cortex, we aim to develop a spiking } \\
& \text { neural network model that enables us to understand network mechanisms of observed experi- } \\
& \text { mental findings and relates structural and functional connectivity. }
\end{aligned}
$$

\section{Experimental Resting State Data}

Massively parallel spiking data were recorded from pre-/motor cortex of an awake macaque monkey at rest, i.e. while the animal was not involved in any task nor received controlled stimuli. The monkey's befa wer was video recorded and reveled periods with and without spontas - two macaque monkeys

- $4 \times 4 \mathrm{~mm}^{2} 10 \times 10$ electrodes Utah Array

- layer 4-5 of monkey motor cortex

-15-20 min registration accompanied by video recordin

- approx. 140 single units per monkey after spike sorting

Preprocessing

- Based on their spike widths, single units were classfied as putative excitatory (exc) or inhibitory (inh).

- Only resting state (RS) periods were extracted

from the data and cut into $5 \mathrm{~s}$ slices for the con-

parison.

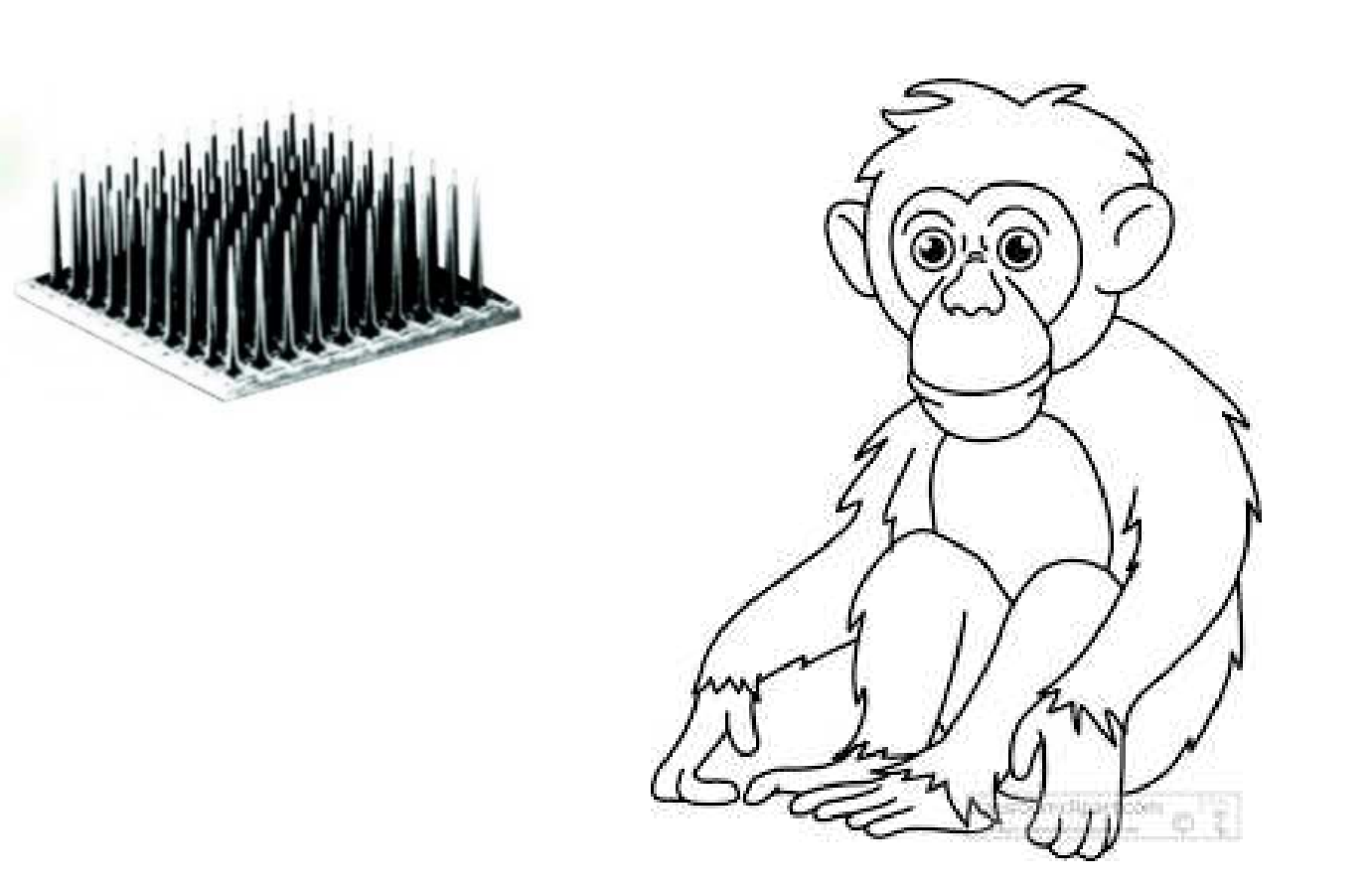

\section{Cortical Network Model}

The simulated network is based on the generic layered cortical microcircuit [1], simulated usin NEST [2]. To enable comparison with the experimental data, the model was extended [3] to

- $\sim 1.2$ million leaky integrate-and-fire neurons in 4 layers with excitatory (E) and inhibitory (I) populations

5.5 billion static current-based synapses - external input with Poisson statistics

- uniform neuron distribution with periodic boundary conditions

- connection probabilities derived from experimental data [1]

- distance-dependent connectivity with Gaussian profile

Preprocessing

- Spiking activity of layer 4 (L4) and layer 5 (L5) was extracted.

- The recorded model neurons were subsampled to match the numbers of excitatory and inhibitory single units in the experiment.

References

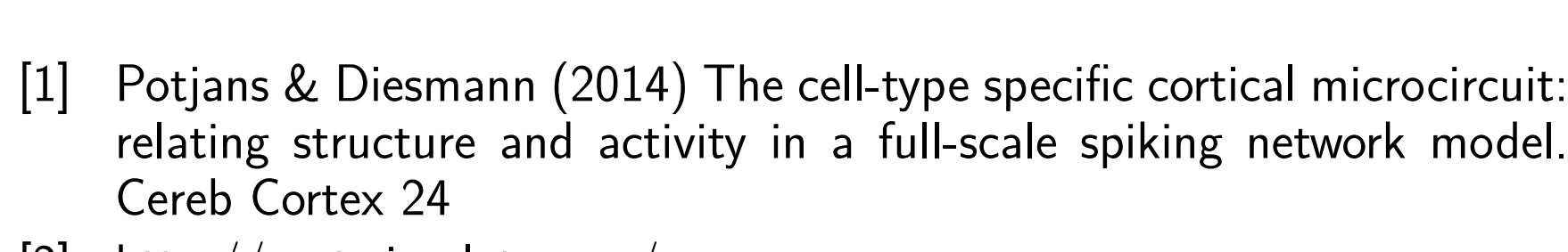

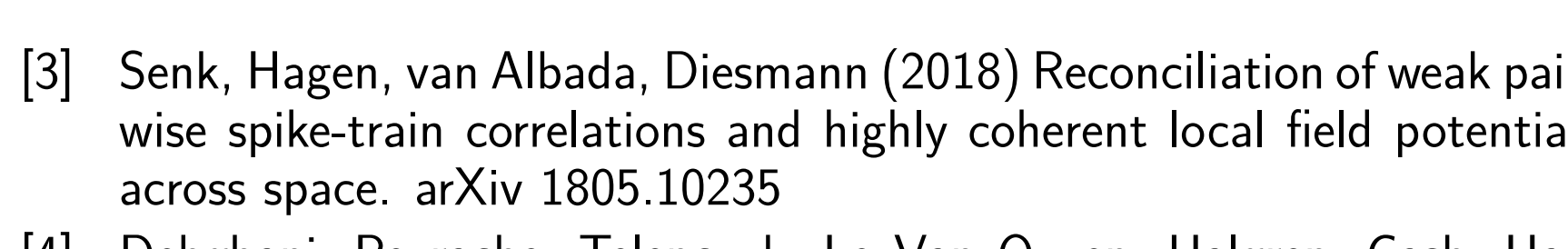

\section{Spiking activity}

The raster plots of an example $10 \mathrm{~s}$ observation (middle figure) of simulated and experimental activity reveal that although population spike counts are similar (top histogram), the average firing rates (FR) tend to be more homogeneous in the simulated results (meso $L 5$ and meso $L 4$, bar plot on the right side of raster plot). This is shown in detail in the FR distributions on the right (mean FR per unit, $5 \mathrm{~s}$ slices), which are narrower
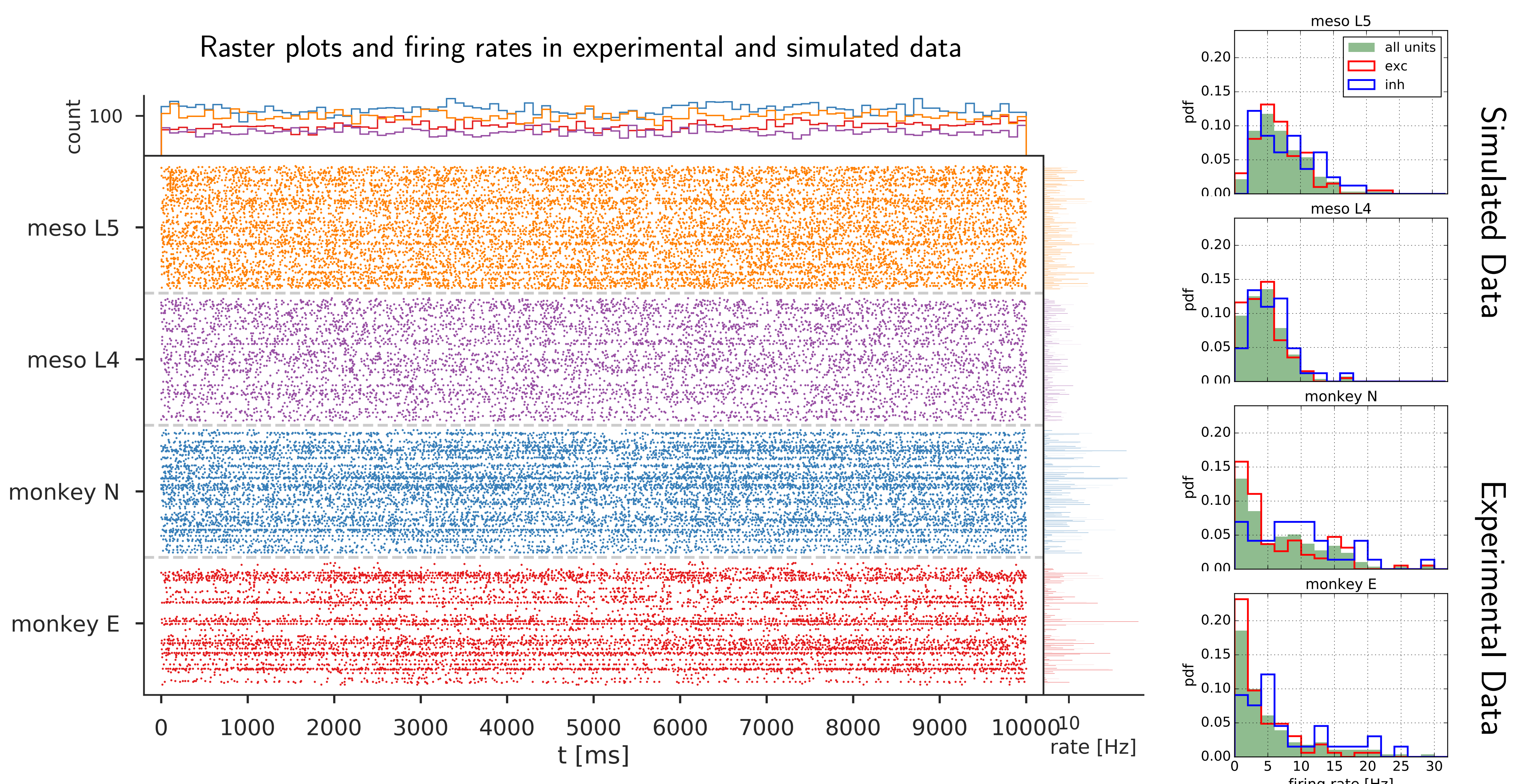

\section{Pairwise Fine Correlations}

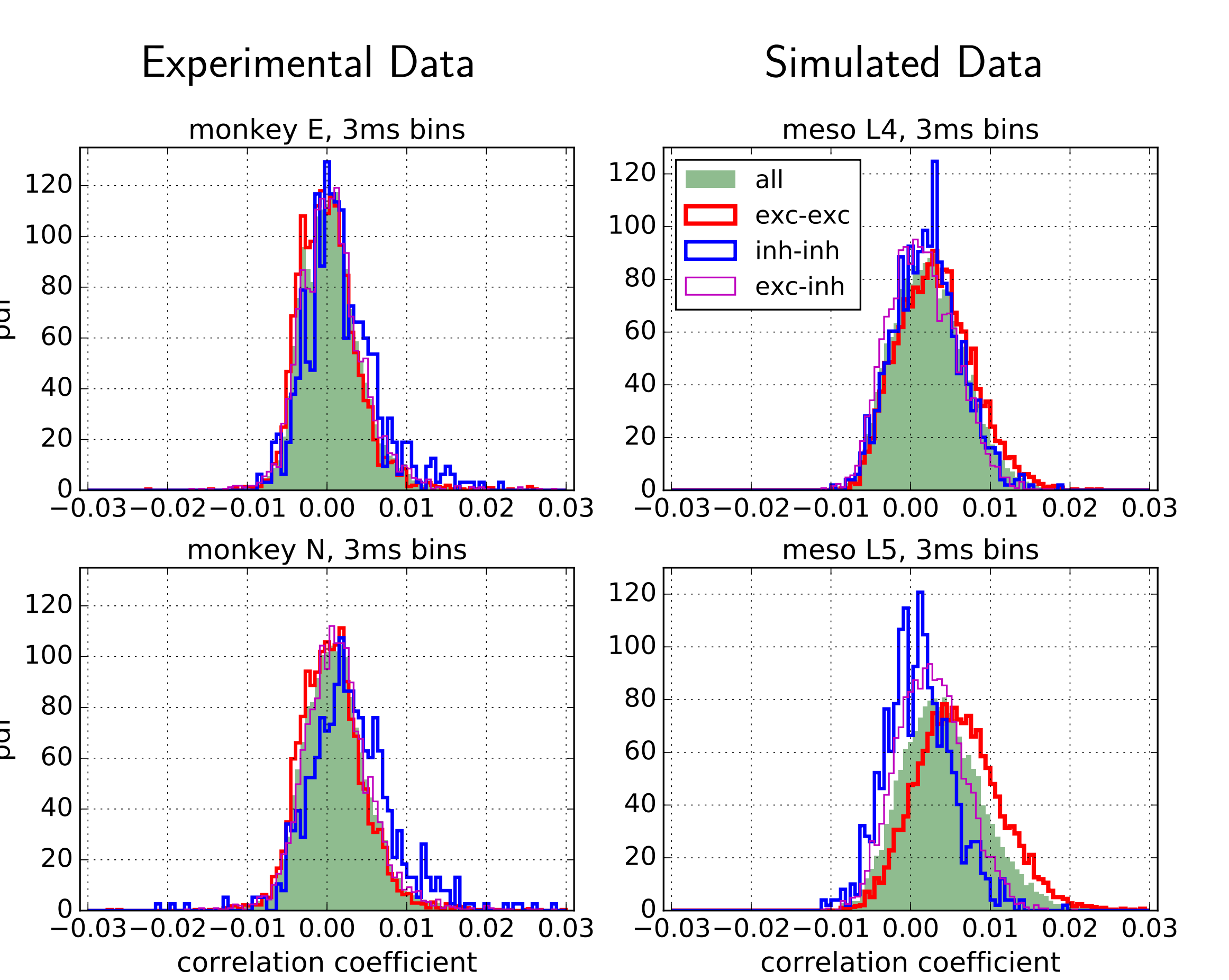

Pairwise fine temporal correlation (CC) between single units can be used to estimate synchrony in a scale characteristic for single action potentials. Therefore only coincidential spikes contribute to the resulting statistics.

- Within each dataset distributions for exc and inh pairs differ between each other.

- Mean CC of experimental data is higher for inh compared to exc units and vice versa for model

Mesocircuit results seem to be less skewed than experimental distribution, which has a larger positive tail.

\section{$\int \underset{\text { Jorschungszentrum }}{\text { ÜLH }}$}

\section{RWWHACHEN JARA A}

(Aix*Marseille

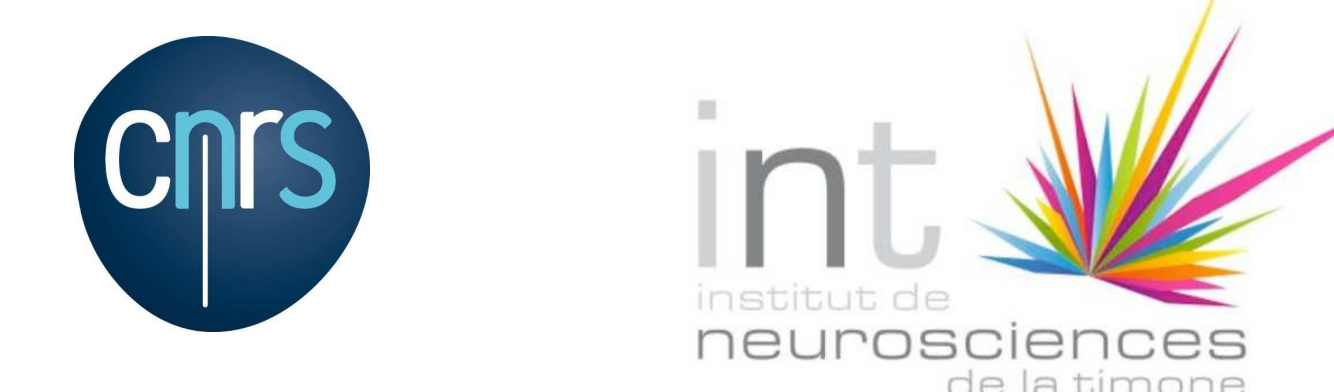

\section{Pairwise Covariances}

Pairwise cross-covariances (COV) calculated with $200 \mathrm{~ms}$ bins reveal co-modulation of firing rates between examined spike trains. Non-stationarities, e.g. abrupt changes of FR, can lead to asymmetric COV distributions with large positive tails.

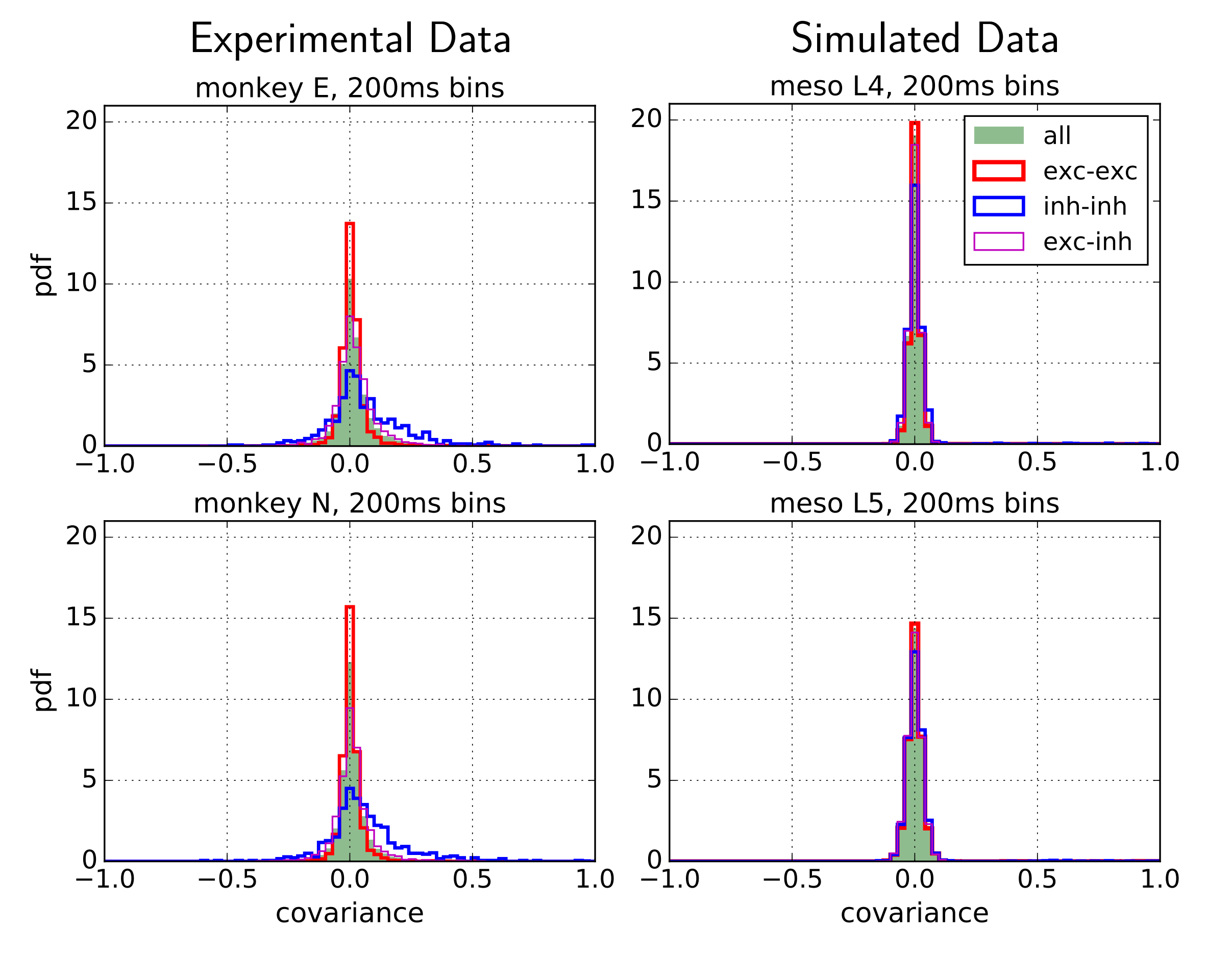

- Putative excitatory and inhibitory distributions differ significantly in experimental data, but not in the model, both average value and shape.

kably similar in all datasets. - Only experimentally obtained COV distributions for putative inhibitory pairs show a pronounced positive tail (asymmetry).

\section{Summary}

- Spiking activity statistics show similar population counts but different average FR per unit distributions for simulated and experimental results.

- Paimise measures reveal asymetries pronounced in experimental inh pairs and virtually absent in simulated data.

- Connectivity parameters used in the model are derived from varion motor cortex activites. Resting state, often described as a superposition of multiple brain states, may be notably less homogen

Uutlook: succesive adaptation of the model connectivity to values specific to monkey motor cortex, until experimental and simulation statistics agree. 